[5] M. A. Dahleh and J. B. Pearson, "Minimization of a regulated response to a fixed input," IEEE Trans. Automat. Contr., vol. 33, pp. 924-930, 1988.

[6] G. Deodhare and M. Vidyasagar, "Control system design via infinite linear programming," Int. J. Contr., vol. 55, no. 6, pp. 1351-1380, 1992.

[7] R. D. Hill and M. E. Halpern, "Minimum overshoot design for SISO discrete-time systems," IEEE Trans. Automat. Contr., vol. 38, pp. 155-158, Jan. 1993.

[8] V. Kucěra, Discrete Linear Control. New York: Wiley, 1979.

[9] D. G. Luenberger, Optimization by Vector Space Methods. New York: Wiley, 1969.

[10] K. L. Moore and S. P. Bhattacharyya, "A technique for choosing zero locations for minimal overshoot," IEEE Trans. Automat. Contr., vol. 35 pp. 577-580, May 1990.

[11] S. Stiefel, "Note on Jordan elimination, linear programming and Tchebycheff approximation," Numerische Mathematik, vol. 2, pp. 1-17, 1960

[12] D. C. Youla, J. J. Bongiorno, and H. A. Jabr, "Modern Wiener-Hopf design of optimal controllers-Part I," IEEE Trans. Automat. Contr., vol. 21 , pp. $3-15,1976$

\section{Risk-Sensitive Filtering and Smoothing via Reference Probability Methods}

\author{
Subhrakanti Dey and John B. Moore
}

\begin{abstract}
In this paper, we address the risk-sensitive filtering problem which is minimizing the expectation of the exponential of the squared estimation error multiplied by a risk-sensitive parameter. Such filtering can be more robust to plant and noise uncertainty than minimum error variance filtering. Although optimizing a differently formulated performance index to that of the so-called $H_{\infty}$ filtering, risk-sensitive filtering leads to a worst case deterministic noise estimation problem given from the differential game associated with $H_{\infty}$ filtering. We consider a class of discrete-time stochastic nonlinear state-space models. We present linear recursions in the information state and the result for the filtered estimate that minimizes the risk-sensitive cost index. We also present fixed-interval smoothing results for each of these signal models. In addition, a brief discussion is included on relations of the risk-sensitive estimation problem to minimum variance estimation and a worst case estimation problem in a deterministic noise scenario related to minimax dynamic games.

The technique used in this paper is the so-called reference probability method which defines a new probability measure where the observations are independent and translates the problem to the new measure. The optimization problem is solved using simple estimation theory in the new measure, and the results are interpreted as solutions in the original measure.
\end{abstract}

Index Terms-Estimation theory, optimal filtering, smoothing.

\section{INTRODUCTION}

Optimal linear stochastic estimation theory, which is known as Kalman filtering theory, has been dominant for the past two decades. In application to Gauss-Markov systems, it achieves the conditional mean estimate, being at the same time the minimum variance estimate and indeed also the maximum-likelihood estimate [1]. The term minimum variance estimate implies the minimization of the energy

Manuscript received April 25, 1996; revised October 22, 1996. This work was supported in part by the Cooperative Research Centre for Robust and Adaptive Systems by the Australian Commonwealth Government under the Cooperative Research Centres Program.

The authors are with the Cooperative Research Center for Robust and Adaptive Systems, Department of Systems Engineering, Research School of Information Sciences and Engineering, Australian National University, Canberra ACT 0200, Australia.

Publisher Item Identifier S 0018-9286(97)07636-8. of the estimation error, or the squared filtering error. Of course, minimum variance estimation can be achieved also for nonlinear stochastic systems via infinite-dimensional filters in general. A more general estimation problem is minimizing the exponential of the squared filtering error, or its expectation, thus penalizing all the higher order moments of the estimation error energy. This problem is termed the risk-sensitive filtering problem, in analogy with a corresponding risk-sensitive control problem. Risk-sensitive filtering also makes a connection with the so-called $H_{\infty}$ filtering problem. The index of the exponential is usually weighted by a risk-sensitive parameter which exaggerates the error when the risk is high due to plant and noise uncertainty, so that risk-sensitive filtering allows a tradeoff between optimal filtering for the nominal model case and the average noise situation and robustness to worst case noise and model uncertainty. When this risk-sensitive parameter approaches zero, the filter is the optimal $L_{2}$ filter, termed here risk-neutral filter.

The risk-sensitive filtering problem has been addressed for linear Gauss-Markov signal models in [2]. The optimizing estimate is derived from a linear filter. In fact, it is an $H_{\infty}$ filter. Off-line Riccati equations are solved to achieve the filter gain which becomes the so-called Kalman gain when the risk-sensitive parameter approaches zero. Risk-sensitive control problems are relatively more abundant in the literature [5], [7], [8]. A solution to the output feedback problem for linear and nonlinear discrete-time systems using information state techniques has been proposed in [9] and [4]. Also, tracking problems for the linear, exponential, quadratic index case have been solved in [4]. The feedback and feedforward gains for the information state in this case require the solution of a backward Riccati and forward linear difference equation, analogous to the standard Linear Quadratic Gaussian (LQG) tracking problem solution. The derivation techniques are based on a reference probability method. The risk-sensitive filtering problem is similar in nature to its control counterpart, and it makes sense to ask whether there are corresponding nonlinear stochastic risk-sensitive filtering results, perhaps dualizing the risk-sensitive control results. Instead of solving a backward dynamic programming recursion to obtain a sequence of admissible controls, we can, at each time point, calculate the filtered estimates recursively in the forward direction based on the observations available to that point. This is a more natural approach than the backward dynamic programming approach taken in [2]. Risk-sensitive control problems are closely connected to minimax dynamic games. Such relations have been explored in [6] and [9] in the small noise limit case. Similarly, risk-sensitive filtering problems are closely connected with the $\mathrm{H}_{\infty^{-}}$ filtering theory developed in [11] and [12]. This relationship has been explored for general nonlinear signal models in a follow-up paper to the present one [15]. It has been shown that in the small noise limit, risk-sensitive filters have an interpretation in terms of a deterministic worst case noise estimation problem given from a differential game.

In this paper, we consider a class of stochastic nonlinear statespace signal models, involving information states, and derive, in the first instance, information state filters based on the risk-sensitive cost index. These filters are linear and infinite-dimensional. The optimizing estimate is then given as the minimizing argument of a particular integral involving the information state. Backward filters and fixed-interval smoothing results are obtained as well. Risksensitive filtering and smoothing results for Hidden Markov Models (HMM's) with finite-discrete states are given in a companion paper [17]. The results in the present paper have been specialized for linear Gauss-Markov systems in [16]. 
The derivation techniques used in this paper are different than the ones used for earlier filtering results in [2], but similar to those used for the control results in [9] and [10]. They are based on a discrete-time version of the well-known change of probability measure technique which has been used in continuous-time nonlinear filtering, a good exposition of which can be found in [18]. Developed in [3], this technique is based on a discrete-time version of Girsanov's theorem, Kolmogorov's Extension theorem, and Fubini's theorem. The preliminary task is to define a new probability measure where the observations are independently identically distributed (i.i.d) and also independent of the state process. Then, one can reformulate the optimization problem in the new measure to obtain the recursions in the information state, the expression for the optimizing filtered estimate, and also density functions of the smoothed estimates by using and exploiting the independence of the observations. Solving the problem in the new measure is equivalent to solving the problem in the old measure as long as a restriction is set on a certain Radon-Nikodym derivative described. Moreover, it is shown that known risk-neutral filtering results can be recovered from the risksensitive results as a special case when the risk-sensitive parameter tends to zero.

In Section II, we describe a nonlinear stochastic state-space model, formally define the risk-sensitive filtering problem, and then deal with the change of measure and reformulation of the problem in the new probability measure to achieve the filtering and smoothing results. Section III briefly discusses the connection of the risk-sensitive estimation problem to risk-neutral (minimum variance) filtering and a worst case noise estimation problem given from a differential game in the small noise limit. Section IV presents some concluding remarks.

\section{FILTERING AND SMOOTHING FOR NONLINEAR Discrete-Time State-Space Models}

In this section, we consider a nonlinear stochastic state-space model. We first introduce the measure change technique and reformulate the problem in the new probability measure. Next, we present an infinite-dimensional linear recursion for the information state and express the optimizing state estimate in terms of an integral involving this information state. Finally, smoothing results are presented.

\section{A. Signal Model}

We consider the following discrete-time state-space model defined on a probability space $(\Omega, \mathcal{F}, P)$ :

$$
\begin{aligned}
x_{k+1} & =A\left(x_{k}\right)+w_{k+1} \\
y_{k} & =C\left(x_{k}\right)+v_{k}
\end{aligned}
$$

where $w_{k} \in \mathbb{R}^{n}, v_{k} \in \mathbb{R}^{p}, x_{k} \in \mathbb{R}^{n}$, and $y_{k} \in \mathbb{R}^{p}$. Here, $x_{k}$ denotes the state of the system, $y_{k}$ denotes the measurement, $w_{k}$ and $v_{k}$ are the process noise and measurement noise, respectively. The vectors $A\left(x_{k}\right)$ and $C\left(x_{k}\right)$ have entries which, in general, are nonlinear functions of $x_{k}$ and $k \in\{0,1, \cdots, T\}$. We assume $A: \mathbb{R}^{n} \rightarrow \mathbb{R}^{n}, C: \mathbb{R}^{n} \rightarrow \mathbb{R}^{p}$ are measurable functions. We also assume that $w_{k}, k \in \mathbb{N}$ has a density function $\psi$, and $v_{k}, k \in \mathbb{N}$ has a strictly positive density function $\phi$. The initial state $x_{0}$ or its density is assumed to be known, and $w_{k}$ is independent of $v_{k}$.

Remark 2.1: Note that one can consider a more general class of nonlinear systems (i.e., with nonlinearities in the noise variables as well). We would just like to comment here that under some conditions, we can obtain risk-sensitive filtering and smoothing results for such generalized nonlinear signal models using the techniques of this paper.

\section{B. Problem Definition}

Define $X_{k} \triangleq\left\{x_{0}, x_{1}, \cdots, x_{k}\right\}, Y_{k} \triangleq\left\{y_{0}, y_{1}, \cdots, y_{k}\right\}$, the $\sigma$-field generated by $Y_{k}$ as $\mathcal{Y}_{k}^{0}$ and the $\sigma$-field generated by $X_{k}$ and $Y_{k-1}$ by $\mathcal{G}_{k}^{0}$. The corresponding complete filtrations are denoted as $\mathcal{Y}_{k}$ and $\mathcal{G}_{k}$, respectively. We define $\hat{x}_{t \mid t}$ as the estimate of the state $x_{t}$ given $\mathcal{Y}_{t}$ and work with recursive estimates which update $\hat{x}_{t \mid t}$ from knowledge of $\hat{x}_{k-1 \mid k-1}, k=1,2, \cdots, t$.

Now, recall that the minimum variance estimate is defined by

$$
\begin{aligned}
\hat{x}_{t \mid t}=\underset{\xi \in \mathbb{R}^{r^{2}}}{\arg \min } E\left[\frac{1}{2}\left(x_{t}-\xi\right)^{\prime} Q\left(x_{t}-\xi\right) \mid \mathcal{Y}_{t}\right] \\
=\underset{\xi \in \mathbb{R}^{n^{\prime}}}{\arg \min } E\left[\frac { 1 } { 2 } \left\{\sum_{k=1}^{t-1}\left(x_{k}-\hat{x}_{k \mid k}\right)^{\prime} Q\left(x_{k}-\hat{x}_{k \mid k}\right)\right.\right. \\
\left.\left.+\left(x_{t}-\xi\right)^{\prime} Q\left(x_{t}-\xi\right)\right\} \mid \mathcal{Y}_{t}\right]
\end{aligned}
$$

where $Q \geq 0$. The equivalence of these two optimization tasks is well known and follows from the linearity property of the expectation operator. One risk-sensitive generalization of this problem that is perhaps very apparent is to find $\hat{x}_{t \mid t}$ such that

$$
\hat{x}_{t \mid t} \in \arg \min _{\xi \in \mathbb{R}^{n 2}} E\left[\exp \left\{\frac{\theta}{2}\left(x_{t}-\xi\right)^{\prime} Q\left(x_{t}-\xi\right)\right\} \mid \mathcal{Y}_{t}\right]
$$

where $\theta>0$ is the risk-sensitive parameter. This generalizes the first optimization task of (2) to the risk-sensitive case. This problem has been solved for continuous-time and discrete-time nonlinear and linear signal models in [15]. It has been shown there that for the linear Gaussian signal model, this is identical to the minimum variance estimate or the Kalman filter.

We concentrate on a related risk-sensitive estimation problem which has been solved for the linear Gaussian signal model in [2]. The problem objective is to determine an estimate $\hat{x}_{t \mid t}$ of $x_{t}$ such that

$$
\hat{x}_{t \mid t} \in \arg \min _{\zeta} J_{t}(\zeta), \quad \forall t=0,1, \cdots, T
$$

where

$$
J_{t}(\zeta)=E\left[\exp \left(\theta \Psi_{0, t}(\zeta)\right) \mid \mathcal{Y}_{t}\right]
$$

is the risk-sensitive cost function. Here

$$
\Psi_{0, t}(\zeta)=\hat{\Psi}_{0, t-1}+\frac{1}{2}\left(x_{t}-\zeta\right)^{\prime} Q_{t}\left(x_{t}-\zeta\right)
$$

where

$$
\hat{\Psi}_{m, n}=\frac{1}{2} \sum_{k=m}^{n}\left(x_{k}-\hat{x}_{k \mid k}\right)^{\prime} Q_{k}\left(x_{k}-\hat{x}_{k \mid k}\right) .
$$

Assume $Q_{k}>0$. This risk-sensitive index generalizes the second optimization task of (3) to the risk-sensitive case.

Remark 2.2: Note that a more general convex cost function $L\left(x_{k}, \hat{x}_{k \mid k}\right)$ can be considered in the index of exponential in (6), instead of the usual quadratic cost as given in (7), as long as $L\left(x_{k}, \hat{x}_{k \mid k}\right)$ is quadratically upper bounded. Similar techniques as presented in this paper can be applied to obtain risk-sensitive filtering and smoothing results for such a cost index. Such a cost index can be useful when the noise distributions are non-Gaussian or we want to obtain finite-dimensional risk-sensitive filters for discrete-time nonlinear systems (see [23]).

Remark 2.3: Note also that the above optimization task given by (6) and (7) bases the minimization over the required risk-sensitive estimate $\hat{x}_{k \mid k}$ at only one time point $k$, assuming knowledge of all the previous estimates. This is preferred over (4) because it results in an $H_{\infty}$ filter for a linear system and a worst case noise estimation problem given from a differential game in general nonlinear cases. 
One could, of course, define the risk-sensitive estimation problem more like an optimal risk-sensitive control problem and use backward dynamic programming to solve for all the risk-sensitive filtered estimates up until time $k$, based on observations up until time $k$. In that case, the estimation problem becomes a combined smoothing and filtering problem and is no longer a pure filtering problem. Therefore, we base the analysis of this paper on the optimization problem as given by (6) and (7), posing it as a forward dynamic programming problem.

\section{Change of Measure}

Define

$$
\begin{aligned}
& \bar{\lambda}_{k}=\frac{\phi\left(y_{k}-C\left(x_{k}\right)\right)}{\phi\left(y_{k}\right)} \\
& \bar{\Lambda}_{k}=\Pi_{l=0}^{k} \bar{\lambda}_{l} .
\end{aligned}
$$

A new probability measure $\bar{P}$ can be defined where $y_{l}, l \in \mathbb{N}$ are independent with density functions $\phi$ and the dynamics of $x$ are as under $P$.

By setting the restriction on the Radon-Nikodym derivative $\left.(d P / d \bar{P})\right|_{\mathcal{G}_{k}}=\bar{\Lambda}_{k}$, the measure $P$ can be defined starting with $\bar{P}$. The existence of $P$ follows from Kolmogorov's Extension theorem [3].

Lemma 2.1: Under $P$, the $\left\{v_{l}\right\}, l \in \mathbb{N}$, are i.i.d. having densities $\phi$.

Proof: The proof is easy and can be achieved by similar techniques as in [3].

\section{Reformulated Cost Criteria}

In this section, we will work under measure $\bar{P}$, where $y_{k}, k \in \mathbb{N}$ is a sequence of independent real random variables with densities $\phi$ and $x_{k+1}=A\left(x_{k}\right)+w_{k}$, where $w_{k}, k \in \mathbb{N}$ are independent random variables with densities $\psi$.

From a version of Bayes' theorem, our cost-index becomes

$$
E\left[\exp \left(\theta \Psi_{0, t}(\zeta)\right) \mid \mathcal{Y}_{t}\right]=\frac{\left.\bar{E}\left[\bar{\Lambda}_{t} \exp \left(\theta \Psi_{0, t}(\zeta)\right)\right) \mid \mathcal{Y}_{t}\right]}{\bar{E}\left[\bar{\Lambda}_{t} \mid \mathcal{Y}_{t}\right]}
$$

where $\bar{E}$ denotes expectation under $\bar{P}$. Hence, our problem objective becomes to determine an $\hat{x}_{t \mid t}$ such that

$$
\hat{x}_{t \mid t}=\underset{\zeta}{\arg \min } \bar{E}\left[\bar{\Lambda}_{t} \exp \left(\theta \Psi_{0, t}(\zeta)\right) \mid \mathcal{Y}_{t}\right] .
$$

\section{E. Recursive Estimates}

Definition 2.1: Define $\alpha_{k \mid k-1}(x)$ as the unnormalized density function such that

$$
\alpha_{k \mid k-1}(x) d x=\bar{E}\left[\bar{\Lambda}_{k-1} \exp \left(\theta \hat{\Psi}_{0, k-1}\right) I\left(x_{k} \in d x\right) \mid \mathcal{Y}_{k-1}\right] .
$$

Remark 2.4: Note that $\alpha_{k \mid k-1}(x)$ can also be interpreted as an information state [26]. It can be considered as the information state of an augmented plant where the state includes the actual state of the system and part of the risk-sensitive cost. For details and the outline of an alternative treatment of our problem, see [15].

Lemma 2.2: The information state $\alpha_{k}(x)$ obeys the following recursion:

$$
\begin{aligned}
\alpha_{k+1 \mid k}(x)= & \frac{1}{\phi\left(y_{k}\right)} \int_{\mathbb{R}^{n}} \phi\left(y_{k}-C(z)\right) \\
& \cdot \exp \left(\frac{1}{2} \theta\left(z-\hat{x}_{k \mid k}\right)^{\prime} Q_{k}\left(z-\hat{x}_{k \mid k}\right)\right) \\
& \cdot \psi(D(x, z)) \alpha_{k \mid k-1}(z) d z .
\end{aligned}
$$

Proof: Suppose $f: \mathbb{R}^{n} \rightarrow \mathbb{R}$ is any Borel test function. Then, using Definition 2.1, we have

$$
\begin{aligned}
& \bar{E}\left[f\left(x_{k+1}\right) \bar{\Lambda}_{k} \exp \left(\theta \hat{\Psi}_{0, k}\right) \mid \mathcal{Y}_{k}\right] \\
& =\int_{\mathbb{R}^{n}} f(\xi) \alpha_{k+1 \mid k}(\xi) d \xi \\
& =\bar{E}\left[f\left(x_{k+1}\right) \bar{\lambda}_{k} \exp \left(\theta \hat{\Psi}_{k, k}\right) \bar{\Lambda}_{k-1} \exp \left(\theta \hat{\Psi}_{0, k-1}\right) \mid \mathcal{Y}_{k}\right] \\
& =\bar{E}\left[f\left(A\left(x_{k}\right)+w_{k+1}\right) \frac{\phi\left(y_{k}-C\left(x_{k}\right)\right)}{\phi\left(y_{k}\right)}\right. \\
& \cdot \exp \left(\frac{1}{2} \theta\left(x_{k}-\hat{x}_{k \mid k}\right)^{\prime} Q_{k}\left(x_{k}-\hat{x}_{k \mid k}\right)\right) \\
& \left.\cdot \bar{\Lambda}_{k-1} \exp \left(\theta \hat{\Psi}_{0, k-1}\right) \mid \mathcal{Y}_{k}\right] \\
& =\bar{E}\left[\int_{\mathbb{R}^{n}} f\left(A\left(x_{k}\right)+w\right) \frac{\phi\left(y_{k}-C\left(x_{k}\right)\right)}{\phi\left(y_{k}\right)}\right. \\
& \cdot \exp \left(\frac{1}{2} \theta\left(x_{k}-\hat{x}_{k \mid k}\right)^{\prime} Q_{k}\left(x_{k}-\hat{x}_{k \mid k}\right)\right) \\
& \left.\cdot \bar{\Lambda}_{k-1} \exp \left(\theta \hat{\Psi}_{0, k-1}\right) \psi(w) d w \mid \mathcal{Y}_{k-1}\right] \\
& \left.=\frac{1}{\phi\left(y_{k}\right)} \int_{\mathbb{R}^{n}} \int_{\mathbb{R}^{n}} f(A(z)+w)\right) \phi\left(y_{k}-C(z)\right) \\
& \exp \left(\frac{1}{2} \theta\left(z-\hat{x}_{k \mid k}\right)^{\prime} Q_{k}\left(z-\hat{x}_{k \mid k}\right)\right) \\
& \text { - } \psi(w) \alpha_{k \mid k-1}(z) d w d z \\
& =\frac{1}{\phi\left(y_{k}\right)} \int_{\mathbb{R}^{n}} \int_{\mathbb{R}^{n}} f(\xi) \phi\left(y_{k}-C(z)\right) \\
& \cdot \exp \left(\frac{1}{2} \theta\left(z-\hat{x}_{k}\right)^{\prime} Q_{k}\left(z-\hat{x}_{k}\right)\right) \\
& \text { - } \psi(D(\xi, z)) \alpha_{k \mid k-1}(z) d \xi d z
\end{aligned}
$$

where $\xi=A(z)+w$, such that $w=D(\xi, z)=\xi-A(z), z=z$ and $d w d z=|\bar{J}(\xi, z)| d \xi d z$. Here

$$
|\bar{J}(\xi, z)|=|\operatorname{det}| \begin{array}{ll}
\frac{\partial w}{\partial \xi} & \frac{\partial w}{\partial z} \\
\frac{\partial z}{\partial \xi} & \frac{\partial z}{\partial z}
\end{array}||=1 .
$$

Since this identity holds for every Borel test function $f$, we have

$$
\begin{aligned}
\alpha_{k+1 \mid k}(x)= & \frac{1}{\phi\left(y_{k}\right)} \int_{\mathbb{R}^{n}} \phi\left(y_{k}-C(z)\right) \\
& \cdot \exp \left(\frac{1}{2} \theta\left(z-\hat{x}_{k \mid k}\right)^{\prime} Q_{k}\left(z-\hat{x}_{k \mid k}\right)\right) \\
& \cdot \psi(D(x, z)) \alpha_{k \mid k-1}(z) d z .
\end{aligned}
$$

Remark 2.5: Supposing $\pi_{0}(z)$ is the density function of $x_{0}$, so for any Borel set $A \in \mathbb{R}^{n}$, we have $P\left(x_{0} \in A\right)=\bar{P}\left(x_{0} \in\right.$ $A)=\int_{A} \pi_{0}(z) d z$. Then $\alpha_{0 \mid-1}(z)=\pi_{0}(z)$, and all the subsequent estimates follow from Lemma 2.2 .

Remark 2.6: It should be noted here that the recursive information state filter, giving $\alpha_{k \mid k-1}(x)$, in terms of $\alpha_{k-1 \mid k-2}(x)$ is linear and infinite dimensional. It is well known from the literature that finite-dimensional filters for general nonlinear stochastic discrete-time signal models are yet to be found. However, finite-dimensional filters do exist for a class of discrete-time nonlinear systems [24]. Similar finite dimensionality conditions for risk-sensitive filters have been derived in [22]. Also, in [23], it has been shown how one can obtain finite-dimensional risk-sensitive filters and smoothers for discretetime nonlinear systems by considering a generalized risk-sensitive cost index which, when suitably chosen, absorbs the contribution from the nonlinear terms. Similar finite-dimensional control results are obtained in [25]. 
Theorem 2.1: The optimal $\hat{x}_{t \mid t}$ can be expressed as

$$
\begin{gathered}
\hat{x}_{t \mid t} \in \underset{\zeta}{\arg \min } \int_{\mathbb{B}^{n z}} \alpha_{t \mid t-1}(z) \frac{\phi\left(y_{t}-C(z)\right)}{\phi\left(y_{t}\right)} \\
\cdot \exp \left(\frac{1}{2} \theta(z-\zeta)^{\prime} Q_{t}(z-\zeta)\right) d z .
\end{gathered}
$$

Proof:

$$
\begin{aligned}
& \bar{E}\left[\bar{\Lambda}_{t} \exp \left(\theta \Psi_{0, t}(\zeta)\right) \mid \mathcal{Y}_{t}\right] \\
&=\bar{E} {\left[\frac{\phi\left(y_{t}-C\left(x_{t}\right)\right)}{\phi\left(y_{t}\right)} \exp \left(\frac{1}{2} \theta\left(x_{t}-\zeta\right)^{\prime} Q_{t}\left(x_{t}-\zeta\right)\right)\right.} \\
&\left.\cdot \bar{\Lambda}_{t-1} \exp \left(\theta \hat{\Psi}_{0, t-1}\right) \mid \mathcal{Y}_{t-1}\right] \\
&=\int_{\mathbb{B}^{n}} \alpha_{t \mid t-1}(z) \frac{\phi\left(y_{t}-C(z)\right)}{\phi\left(y_{t}\right)} \exp \left(\frac{1}{2} \theta(z-\zeta)^{\prime} Q_{t}(z-\zeta)\right) d z
\end{aligned}
$$

(using the definition of $\alpha_{k \mid k-1}(x)$ ). Using (9), we have (14) which completes the proof.

Remark 2.7: The integrability of the integrands in Lemma 2.2 and Theorem 2.1 has been assumed. A necessary condition for this is that $\theta$ should be sufficiently small. Of course, in the risk-neutral case, the optimal estimate becomes the conditional mean estimate, and its density becomes the conditional probability density. For the linear Gauss-Markov signal model case, the integrability condition simplifies to requiring the existence of the solution of a Riccati equation, which is guaranteed for $\theta$ sufficiently small. See [16] for details.

\section{F. Smoothing}

In this section we obtain the density function of the smoothed state estimates from a fixed set of observations $Y_{T}=\left(y_{0}, \cdots, y_{T}\right)^{\prime}$. We assume knowledge of the optimal filtered estimates $\hat{X}_{T}=$ $\left(\hat{x}_{0 \mid 0}, \cdots, \hat{x}_{T \mid T}\right)^{\prime}$. Using this density, we will then evaluate the smoothed state estimate which optimizes a certain risk-sensitive cost function to be described shortly. This smoothing is essentially an offline processing and technically known as fixed-interval smoothing. We will also define $\hat{X}_{m}^{n}=\left(\hat{x}_{m \mid m}, \cdots, \hat{x}_{n \mid n}\right)$ and $\bar{\Lambda}_{m, n}=\Pi_{k=m}^{n} \bar{\lambda}_{k}$. Now, we will define the unnormalized density of the smoothed estimate $\gamma_{k, T}(x)$ and the backward filtered unnormalized density (or backward information state or reverse likelihood ratio [27]) $\beta_{k, T}(x)$ as follows. We also define the risk-sensitive smoothed estimate $\hat{x}_{k \mid T}^{s}$.

Definition 2.2:

$$
\begin{aligned}
& \gamma_{k, T}(x) d x=\bar{E}\left[\bar{\Lambda}_{T} \exp \left(\theta \hat{\Psi}_{0, T}\right) I\left(x_{k} \in d x\right) \mid \mathcal{Y}_{T}\right] \\
& \beta_{k, T}(x)=\bar{E}\left[\bar{\Lambda}_{k, T} \exp \left(\theta \hat{\Psi}_{k, T}\right) \mid x_{k}=x, \mathcal{Y}_{T}\right] \\
& \hat{x}_{k \mid T}^{s} \in \underset{\xi}{\arg \min E}\left[\exp \left(\theta \hat{\Psi}_{0, T}\right)\right. \\
&\left.\cdot \exp \left\{\frac{\theta}{2}\left(x_{k}-\xi\right)^{\prime} Q_{k}\left(x_{k}-\xi\right)\right\} \mid \mathcal{Y}_{T}\right] \\
& \in \underset{\xi}{\arg \min } \bar{E} {\left[\bar{\Lambda}_{0, T} \exp \left(\theta \hat{\Psi}_{0, T}\right)\right.} \\
& \cdot\left.\exp \left\{\frac{\theta}{2}\left(x_{k}-\xi\right)^{\prime} Q_{k}\left(x_{k}-\xi\right)\right\} \mid \mathcal{Y}_{T}\right]
\end{aligned}
$$

where $Q_{k}>0$.

Lemma 2.3: The process $\beta_{k, T}(x)$ satisfies the following backward recursion:

$$
\begin{aligned}
\beta_{k, T}(x)= & \frac{\phi\left(y_{k}-C(x)\right)}{\phi\left(y_{k}\right)} \exp \left(\frac{\theta}{2}\left(x-\hat{x}_{k \mid k}\right)^{\prime} Q_{k}\left(x-\hat{x}_{k \mid k}\right)\right) \\
& \cdot \int_{\mathbb{R}^{n 2}} \psi(\xi-A(x)) \beta_{k+1, T}(\xi) d \xi
\end{aligned}
$$

where

$$
\beta_{T, T}(x)=\frac{\phi\left(y_{T}-C(x)\right)}{\phi\left(y_{T}\right)} \exp \left(\frac{\theta}{2}\left(x-\hat{x}_{T \mid T}\right)^{\prime} Q_{T}\left(x-\hat{x}_{T \mid T}\right)\right) .
$$

Proof:

$$
\begin{aligned}
& \beta_{k, T}(x)=\bar{E}\left[\bar{\Lambda}_{k, T} \exp \left(\theta \hat{\Psi}_{k, T}\right) \mid x_{k}=x, \mathcal{Y}_{T}\right] \\
& =\bar{E}\left[\bar{\lambda}_{k} \exp \left(\theta \hat{\Psi}_{k, k}\right) \bar{\Lambda}_{k+1, T} \exp \left(\theta \hat{\Psi}_{k+1, T}\right)\right. \\
& \left.\cdot \mid x_{k}=x, \mathcal{Y}_{T}\right] \\
& =\bar{E}\left[\frac{\phi\left(y_{k}-C\left(x_{k}\right)\right)}{\phi\left(y_{k}\right)}\right. \\
& \cdot \exp \left(\frac{\theta}{2}\left(x_{k}-\hat{x}_{k \mid k}\right)^{\prime} Q_{k}\left(x_{k}-\hat{x}_{k \mid k}\right)\right) \\
& \cdot \bar{E}\left[\bar{\Lambda}_{k+1, T} \exp \left(\theta \hat{\Psi}_{k+1, T}\right) \mid x_{k}=x, x_{k+1}, \mathcal{Y}_{T}\right] \\
& \left.\cdot \mid x_{k}=x, \mathcal{Y}_{T}\right] \\
& =\bar{E}\left[\frac{\phi\left(y_{k}-C\left(x_{k}\right)\right)}{\phi\left(y_{k}\right)}\right. \\
& \cdot \exp \left(\frac{\theta}{2}\left(x_{k}-\hat{x}_{k \mid k}\right)^{\prime} Q_{k}\left(x_{k}-\hat{x}_{k \mid k}\right)\right) \\
& \left.\cdot \beta_{k+1, T}\left(x_{k+1}\right) \mid x_{k}=x, \mathcal{Y}_{T}\right] \\
& =\bar{E}\left[\frac{\phi\left(y_{k}-C\left(x_{k}\right)\right)}{\phi\left(y_{k}\right)}\right. \\
& \cdot \exp \left(\frac{\theta}{2}\left(x_{k}-\hat{x}_{k \mid k}\right)^{\prime} Q_{k}\left(x_{k}-\hat{x}_{k \mid k}\right)\right) \\
& \text { - } \left.\beta_{k+1, T}\left(A\left(x_{k}\right)+w_{k+1}\right) \mid x_{k}=x, \mathcal{Y}_{T}\right] \\
& =\frac{\phi\left(y_{k}-C(x)\right)}{\phi\left(y_{k}\right)} \exp \left(\frac{\theta}{2}\left(x-\hat{x}_{k \mid k}\right)^{\prime} Q_{k}\left(x-\hat{x}_{k \mid k}\right)\right) \\
& \cdot \int_{\mathbb{R}^{n}} \psi(\xi-A(x)) \beta_{k+1, T}(\xi) d \xi \text {. }
\end{aligned}
$$

Theorem 2.2: The unnormalized density function of the smoothed estimate $\gamma_{k, T}(x)$ can be expressed as

$$
\gamma_{k, T}(x)=\alpha_{k \mid k-1}(x) \beta_{k, T}(x) .
$$

Proof: Suppose $f: \mathbb{R}^{n} \rightarrow \mathbb{R}$ is a Borel test function. Then we can write

$$
\begin{aligned}
\bar{E}\left[\bar{\Lambda}_{T}\right. & \left.\exp \left(\theta \hat{\Psi}_{0, T}\right) f\left(x_{k}\right) \mid \mathcal{Y}_{T}\right] \\
= & \int_{\mathbb{B}^{n 2}} f(x) \gamma_{k, T}(x) d x \\
= & \bar{E}\left[\bar{\Lambda}_{k-1} \exp \left(\theta \hat{\Psi}_{0, k-1}\right) f\left(x_{k}\right) \bar{\Lambda}_{k, T} \exp \left(\theta \hat{\Psi}_{k, T}\right) \mid \mathcal{Y}_{T}\right] \\
= & \bar{E}\left[\overline { \Lambda } _ { k - 1 } \operatorname { e x p } ( \theta \hat { \Psi } _ { 0 , k - 1 } ) f ( x _ { k } ) \overline { E } \left[\bar{\Lambda}_{k, T} \exp \left(\theta \hat{\Psi}_{k, T}\right)\right.\right. \\
& \left.\left.\quad \cdot \mid x_{k}, \mathcal{Y}_{T}\right] \mid \mathcal{Y}_{T}\right] \\
= & \left.\bar{E}_{k-1} \exp \left(\theta \hat{\Psi}_{0, k-1}\right) f\left(x_{k}\right) \beta_{k, T}\left(x_{k}\right) \mid \mathcal{Y}_{T}\right] \\
= & \int_{\mathbb{B}^{n}} f(x) \alpha_{k \mid k-1}(x) \beta_{k, T}(x) d x
\end{aligned}
$$

(by using Definition 2.1 and the conditional independence property arising from the fact that $\left\{y_{k}\right\}$ is a sequence of i.i.d. random variables under $\bar{P}$ ). Since $f$ is an arbitrary Borel test function, we have

$$
\gamma_{k, T}(x)=\alpha_{k \mid k-1}(x) \beta_{k, T}(x) .
$$

Remark 2.8: A similar representation of the conditional distribution of the smoothed estimate for continuous-time systems (in the minimum variance or risk-neutral context) in terms of a product of two processes can be found in [19]. 
by

Theorem 2.3: The risk-sensitive smoothed estimate $\hat{x}_{k \mid T}^{s}$ is given

$\hat{x}_{k \mid T}^{s} \in \underset{\xi}{\arg \min } \int_{\mathbb{R}^{n}} \gamma_{k, T}(z) \exp \left(\frac{1}{2} \theta(z-\xi)^{\prime} Q_{k}(z-\xi)\right) d z$.

Proof: The proof is similar to that of Theorem 2.1.

\section{LIMITING RESULTS}

In this section, we briefly discuss the relation of risk-sensitive estimation to minimum variance or risk-neutral estimation and a worst case noise estimation problem given from a differential game connected to $H_{\infty}$ estimation theory. Before proceeding further, we need to restate the optimization problem defined by (5)-(7) in terms of the following equivalent formulation:

$$
\hat{x}_{t \mid t} \in \underset{\zeta}{\arg \min } \frac{\epsilon}{\mu} \log E\left[\exp \left(\frac{\mu}{\epsilon} \Psi_{0, t}(\zeta)\right) \mid \mathcal{Y}_{t}\right]
$$

We also assume that the noise variables $w_{k}, v_{k}$ in (1) are scaled by $\sqrt{\epsilon}$. Note that here $\theta$ has been replaced by $\mu / \epsilon$.

Following a similar treatment in [6] for a risk-sensitive control problem, it can be easily shown that as $\mu \rightarrow 0$, the cost defined by (22) approaches the corresponding risk-neutral cost. It is also obvious from (11) that as $\theta \rightarrow 0$, the recursion approaches the corresponding recursion for a conditional density prediction filter for the signal model (1).

The other interesting case is when $\epsilon \rightarrow 0$, which is known as the small noise limit. It has been shown in [15] that in the small noise limit, the risk-sensitive estimation problem approaches a worst case estimation problem in a deterministic noise scenario given from a differential game. These results have been obtained for both continuous-time and discrete-time systems. Indeed, it is true that for a discrete-time linear Gauss-Markov system, the risk-sensitive filter is an $H_{\infty}$ filter (see [2] or [16] for details). Similar results exist for risk-sensitive control problems [9], [6].

\section{CONCLUSION}

The problem of discrete-time filtering and smoothing with an exponential quadratic error cost-criteria, termed risk-sensitive filtering and smoothing, has been addressed in this paper using a reference probability method and information state techniques. A new probability measure has been defined where observations are i.i.d., and the reformulated cost-criterion has been minimized to give filtering and smoothing results for a class of discrete-time continuous-range nonlinear stochastic state-space models. Linear but infinite-dimensional recursions have been obtained in the information state. Results for the optimizing risk-sensitive estimate, backward filtered density, and the smoothed estimate along with its density have been obtained as well. A brief discussion on relations of risksensitive estimation problems with $L_{2}$ and $H_{\infty}$ estimation problems has been included.

Stability and convergence analysis for such filters are quite difficult in the general nonlinear context. An attempt has been made to prove the property of exponential forgetting of initial conditions for risksensitive filters and smoothers for HMM's in [21]. Also, subsequent research has shown that such optimizing risk-sensitive filtered and smoothed estimates indeed obtain the property of geometric forgetting of initial conditions at a geometric rate [20]. However, extending these results for general nonlinear signal models remains a topic for further research.

\section{REFERENCES}

[1] B. D. O. Anderson and J. B. Moore, Optimal Filtering. Englewood Cliffs, NJ: Prentice-Hall, 1979.

[2] J. L. Speyer, C. Fan, and R. N. Banavar, "Optimal stochastic estimation with exponential cost criteria," in Proc. 31 st Conf. Decision Contr., Dec. 1992, vol. 2, pp. 2293-2298.

[3] R. J. Elliott, L. Aggoun, and J. B. Moore, Hidden Markov Models: Estimation and Control, Appl. Math. Series. New York: SpringerVerlag, 1994.

[4] I. B. Collings, M. R. James, and J. B. Moore, "An information-state approach to risk-sensitive tracking problems," J. Math. Syst., Estimation, Contr., vol. 6, no. 3, July 1996.

[5] P. Whittle, "Risk-sensitive linear/quadratic/Gaussian control," Adv. Appl. Probability 13, pp. 746-777, 1981.

[6] _ Risk-Sensitive Optimal Control. New York: Wiley, 1990.

[7] A. Bensoussan and J. H. van Schuppen, "Optimal control of partially observable stochastic systems with an exponential-of-integral performance index," SIAM J. Contr. Opt., vol. 23, pp. 599-613, 1985.

[8] D. H. Jacobson, "Optimal stochastic linear systems with exponential performance criteria and their relation to deterministic games," IEEE Trans. Automat. Contr., vol. AC-18, pp. 124-131, Apr. 1973.

[9] M. R. James, J. S. Baras, and R. J. Elliott, "Risk-sensitive control and dynamic games for partially observed discrete-time systems," IEEE Trans. Automat. Contr., vol. 39, pp. 780-792, 1994.

[10] R. J. Elliott, "A general recursive discrete-time filter," J. Appl. Probability, vol. 30, pp. 575-588, 1993.

[11] I. Yaesh and U. Shaked, " $H_{\infty}$ optimal estimation: The discrete-time case," in Proc. 9th Int. Symp. MTNS, Kobe, Japan, June 1991, pp. 261-267.

[12] K. Uchida and M. Fujita, "Finite horizon mixed $H_{2}$ and $H_{\infty}$ estimation," in Proc. Fifth Symp. Dynamic Games, Griementz, Switzerland, June 1992, pp. 504-508.

[13] L. Aggoun, A. Bensoussan, R. J. Elliott, and J. B. Moore, "Finitedimensional quasilinear risk-sensitive control," Syst. Contr. Lett.

[14] A. Segall, "Recursive estimation from discrete-time point processes," IEEE Trans. Inform. Theory, vol. IT-22, no. 4, pp. 422-431, 1976.

[15] J. B. Moore, R. J. Elliott, and S. Dey, "Risk-sensitive generalizations of minimum variance estimation and control," J. Math. Syst., Estimation Contr., to be published.

[16] S. Dey and J. B. Moore, "Risk-sensitive filtering and smoothing via reference probability methods," in Proc. Amer. Contr. Conf., Seattle, June 1995, vol. 1, pp. 129-133.

[17] _ _ "Risk-sensitive filtering and smoothing for hidden Markov models," Syst. Contr. Lett., vol. 25, pp. 361-366, Aug. 1995.

[18] O. Zeitouni, "On the reference probability approach to the equations of nonlinear filtering," Stochastics, vol. 19, pp. 133-149, 1986.

[19] E. Pardoux, Nonlinear Filtering and Stochastic Control, Lecture Notes in Mathematics, 1981, p. 981.

[20] V. Krishnamurthy and S. Dey, "On weak ergodicity of filters for hidden Markov models," to be published.

[21] R. K. Boel, J. B. Moore, and S. Dey, "Geometric convergence of filters for hidden Markov models," in Proc. 34th IEEE Conf. Decision Contr., New Orleans, LA, Dec. 1995, pp. 69-74.

[22] S. Dey and J. B. Moore, "On finite-dimensional risk-sensitive estimation," in Proc. Int. Symp. Signal Processing Its Appl., Gold Coast, Australia, Aug. 1995, to be published.

[23] _ _ "Finite-dimensional risk-sensitive filters and smoothers for discrete-time nonlinear systems," to be published.

[24] J. Levine and G. Pignie, "Exact finite-dimensional filters for a class of nonlinear discrete-time systems," Stochastics, vol. 18, pp. 97-132, 1986.

[25] J. B. Moore and J. S. Baras, "Finite-dimensional optimal controllers for nonlinear plants," Syst. Contr. Lett., vol. 26, pp. 223-230, 1995.

[26] P. R. Kumar and P. Varaiya, Stochastic Systems. Englewood Cliffs, NJ: Prentice-Hall, 1986.

[27] B. D. O. Anderson and I. B. Rhodes, "Smoothing algorithms for nonlinear finite-dimensional systems," Stochastics, vol. 9, pp. 139-165, 1983. 